\title{
Stationary Bed Heating Drying Method
}

National Cancer Institute

\section{Source}

National Cancer Institute. Stationary Bed Heating Drying Method. NCI Thesaurus. Code C112984.

A process that removes water or volatile solvents from a bed of solids by relying on heat transfer from the equipment surface or a hot gas to the solid sample. 